\title{
Tubercular uveitis: Nuggets from Collaborative Ocular Tuberculosis Study (COTS)-1
}

\section{Authors:}

Ilaria TESTI ${ }^{*}$, Rupesh AGRAWAL 2,3,4*, Sarakshi MAHAJAN ${ }^{5}$, Aniruddha AGARWAL ${ }^{6}$, Dinesh Visva GUNASEKERAN ${ }^{2,3}$, Dhananjay RAJE7, Kanika AGGARWAL ${ }^{6}$, Somasheila I MURTHY ${ }^{8}$, Mark WESTCOTT ${ }^{3}$, Soon Phaik CHEE ${ }^{4,9}$, Peter MC CLUSKEY ${ }^{10}$, Su Ling HO${ }^{2}$, Stephen $\mathrm{TEOH}^{2}$, Luca CIMINO ${ }^{11}$, Jyotirmay BISWAS ${ }^{12}$, Shishir NARAIN ${ }^{13}$, Manisha AGARWAL ${ }^{14}$, Padmamalini MAHENDRADAS ${ }^{15}$, Moncef KHAIRALLAH $^{16}$, Nicholas JONES ${ }^{17}$, Ilknur TUGAL-TUTKUN ${ }^{18}$, Kalpana BABU ${ }^{19}$, Soumayava BASU ${ }^{20}$, Ester CARREÑO ${ }^{21}$, Richard LEE ${ }^{22}$, Hassan AL-DHIBI ${ }^{23}$, Bahram BODAGHI ${ }^{24}$, Alessandro INVERNIZZI ${ }^{25}$, Debra A. GOLDSTEIN26, Carl P. HERBORT ${ }^{27}$, Talin BARISANIASENBAUER $^{28}$, Julio J GONZÁLEZ-LÓPEZ ${ }^{29}$, Sofia ANDROUDI ${ }^{30}$, Reema BANSAL ${ }^{6}$, Bruttendu MOHARANA ${ }^{6}$, Simona Degli ESPOSTI ${ }^{3}$, Anastasia TASIOPOULOU², Sengal NADARAJAH ${ }^{2}$, Mamta AGARWAL ${ }^{12}$, Sharanaya ABRAHAM ${ }^{12}$, Ruchi VALA ${ }^{15}$, Ramandeep SINGH ${ }^{6}$, Aman SHARMA ${ }^{31}$, Kusum SHARMA ${ }^{32}$, Manfred ZIERHUT ${ }^{33}$, Robert GRANT ${ }^{34}$ Onn Min KON ${ }^{35}$, Emmett T. Cunningham ${ }^{36}$, John Kempen ${ }^{37,38}$, Quan Dong NGUYEN ${ }^{5}$, Carlos PAVESIO ${ }^{3}$, Vishali GUPTA ${ }^{6}$.

\section{Affiliations}

1. Department of Ophthalmology, University of Padova, Padova, Italy

2. National Healthcare Group Eye Institute, Tan Tock Seng Hospital, Singapore

3. Moorfields Eye Hospital, NHS Foundation Trust, London, United Kingdom

4. Singapore Eye Research Institute, Singapore

5. Byres Eye Institute, Stanford University, Palo Alto, California, United States of America

6. Advanced Eye Centre, Postgraduate Institute of Medical Education and Research (PGIMER), Chandigarh

7. MDS Bioanalytics, India

8. Tej Kohli Cornea Institute, LV Prasad Eye Institute, Kallam Anji Reddy Campus, Hyderabad, India

9. Yong Loo Lin School of Medicine, National University of Singapore, Singapore

10. Department of Clinical Ophthalmology \& Eye Health, Central Clinical School, Save Sight Institute, The University of Sydney, Sydney, Australia

11. Ocular Immunology Unit, Azienda USL IRCCS, Reggio Emilia, Italy

12. Department of Ophthalmology, Sankara Nethralaya, Chennai, India

13. Department of Ophthalmology, Shroff Eye Centre, New Delhi, India

14. Department of Ophthalmology, Dr Shroff's Charity Eye Hospital Daryaganj, New Delhi, India

15. Department of Uveitis and Ocular Immunology, Narayana Nethralaya, Bangalore, India

16. Department of Ophthalmology, Fattouma Bourguiba University Hospital, Faculty of Medicine, University of Monastir, Tunisia

17. Department of Ophthalmology, University of Manchester, Manchester, United Kingdom

18. Istanbul University, Istanbul Faculty of Medicine, Department of Ophthalmology, Turkey

19. Prabha Eye Clinic \& Research centre, Vittala International Institute of Ophthalmology, Bangalore, India

20. LV Prasad Eye Institute, Department of Ophthalmology, Bhubaneswar, India

21. Hospital Universitario Fundacion Jimenez Diaz, Madrid, Spain

22. Bristol Eye Hospital, Department of Ophthalmology, Bristol, United Kingdom

23. King Khaled Eye Specialist Hospital, Department of Ophthalmology, Riyadh, Kingdom of Saudi Arabia

24. DHU SightRestore, Department of Ophthalmology, Sorbonne University, Paris, France

25. Eye Clinic, Department of Biomedical and Clinical Science "L. Sacco", Luigi Sacco Hospital, University of Milan, Italy

26. Northwestern University, Feinberg School of Medicine, Department of Ophthalmology. Chicago, Illinois, USA

27. Centre for Ophthalmic Specialised Care \& University of Lausanne, Lausanne, Switzerland 
28. The Centre for Ocular Inflammation and Infection (OCUVAC), Laura Bassi Centre of Expertise Center of Pathophysiology, Infectiology and Immunology, Medical University of Vienna, Vienna, Austria

29. Ramón y Cajal University Hospital, Department of Ophthalmology, Madrid, Spain

30. Department of Ophthalmology, University of Thessaly, Greece

31. Department of Rheumatology, PGIMER, Chandigarh, India

32. Department of Microbiology, PGIMER, Chandigarh, India

33. Centre of Ophthalmology, Department of Ophthalmology, University of Tuebingen, Germany

34. Faculty of Health, Social Care and Education, Kingston University and St George's, University of London, London, United Kingdom

35. Chest and Allergy Clinic, St Mary's Hospital, Imperial College Healthcare NHS Trust, London, United Kingdom

36. The Francis I. Proctor Foundation, UCSF School of Medicine, San Francisco, California, United States

37. Department of Ophthalmology, Massachusetts Eye and Ear, Harvard Medical School, Boston, Massachusetts, United States

38. MCM Eye Unit, MyungSung Christian Medical Center and MyungSung Medical School, Addis Ababa, Ethiopia

\section{Corresponding author:}

Vishali Gupta, MD

Professor of Ophthalmology,

Advanced Eye Centre,

Post graduate Institute of Medical Education and Research (PGIMER),

Chandigarh, India.

Email: vishalisara@yahoo.co.in

Contact number: +919417565506

\section{Key words:}

Tuberculosis;

Choroiditis;

Collaborative Ocular Tuberculosis Study (COTS);

Anti-tubercular therapy;

Global experts;

Tubercular uveitis

Financial support: The research was partially funded by the National Institute for Health Research (NIHR) Biomedical Research Centre at Moorfields Eye Hospital NHS Foundation Trust and UCL Institute of Ophthalmology.

Disclosures: There is no conflict of interest for any authors.

\section{* Joint First Authors}




\section{Abstract}

Tuberculosis (TB) is a major infection that can affect the eye as first and sole presentation without features of systemic disease. Controversy exists regarding diagnosis and management of tubercular uveitis (TBU), further compounded by regional variations in disease expression. Collaborative Ocular Tuberculosis Study (COTS)-1 aims to address knowledge deficits through collaboration amongst uveitis specialists across the globe by sharing the data of patients with TBU presented at participating centers from January 2004 to December 2014. Data collection was facilitated by a novel method of real-time encrypted web-based data entry allowing regular updates as new data and recommendations become available. Information on clinical features, investigation findings, management and treatment outcomes were reviewed to get an idea about real world scenario. The current review aims to focus on methodology and briefing of published reports from COTS group in COTS-1 study to highlight key messages from this large data. 


\section{Introduction}

Tuberculosis (TB) is the leading cause of death from a single infectious agent resulting in estimated 10.0 million new cases with 1.3 million deaths in $2017 .{ }^{1}$ Thirty high TB burden countries account for $87 \%$ of world's cases, contributing to difficulties in its eradication that is mainly attributed to vulnerable populations and the pandemic of HIV. ${ }^{1}$ Ocular TB is an uncommon form of extra pulmonary disease with highly variable prevalence, ranging from $0.2-2.7 \%$ to $5.6-10.5 \%$ in the reported series of uveitis from different regions of the world, due to large and persistent gaps in detection and treatment. ${ }^{2-5}$ Protean clinical manifestations, mixed ocular tissue involvement, lack of international consensus on the use of investigations for diagnosis and absence of rationalized standard guidelines on therapeutic management potentiate the problem of diagnosing and managing ocular TB or tubercular uveitis (TBU) ${ }^{3-8}$

This comprehensive review aims to highlight the findings described in different reports by the retrospective arm of the Collaborative Ocular Tuberculosis Study (COTS), which is the COTS-1. ${ }^{9-13}$ Objective of the COTS- 1 was to address the uncertainties in the diagnosis and therapeutic management of TBU, determining the global clinical profile of TBU and examining regional variation management practices and therapeutic outcomes.

\section{Methods in COTS-1}

This retrospective multinational multicenter cohort study combined data from 25 international eye care centers, guided by the experience of uveitis specialists around the world. A total of 945 patients diagnosed with TBU were included in the study. Patients who fulfilled the inclusion criteria for TBU (Appendix 1) and received a comprehensive examination to exclude other relevant differential diagnosis were recruited in the study if they completed at least one year of follow-up with availability of detailed ophthalmic examinations recorded in their medical records. Diagnostic criteria for TBU were defined 
through a systematic review of literature and guided by background knowledge of the experts of the study group. ${ }^{5,6}$

An online web-based secure and encrypted platform (Cognito Form, Columbia, South Carolina, USA) was developed to facilitate data collection and multinational clinical research collaboration. The smart form facilitated standardized data entry and omitted patient identifiers. This novel data entry platform was designed to address the heterogeneous nature of the disease. One distinct benefit of a web-based platform over existing excel spread sheet data entry form or other data collection sets was the capability to program it to perform as a smart-form and subsequently export data into an organized excel sheet. The smart form improved the integrity of the data set by minimizing data cleaning and associated human errors.

The smart form also optimized data entry by changing to "prompt only" entry of relevant clinical signs depending on the anatomical classification of uveitis initially reported by the user. This function helped us to streamline the collection of information that was extensive in both breath as well as depth, without overwhelming staff involved in data entry. In addition, the capability to mandate entry of certain information before users could progress to later sections ensures that key data points were collected.

The encrypted nature of the form also protected patient data. Patients were indexed based on study site of origin and a unique patient ID without any patient identifiers. This allowed only individuals with access to the respective hospital records and appointment dates to be able to connect the data collected to individual patients' information.

\section{Statistical methods applied in COTS-1}

In the study data set, the categorical variables were summarized in terms of frequencies and percentages and were compared across groups of interest using Pearson's Chisquare test or Fisher's exact test. Treatment failure criteria met in either eye was 
considered as failure event. The failure rates for different study groups were obtained using non-parametric Kaplan-Meier plots and the statistical significance of difference between the rates was determined using Log-rank test. Such analysis was performed to compare the survival rates of different retinal vasculitis types, various phenotypes of choroidal involvement in TBU etc. based on predefined treatment failure criteria.

Multivariate Cox-proportional Hazard analysis was performed to obtain crude and adjusted hazard rates for each clinical sign as an estimate of risk along with $95 \% \mathrm{Cl}$.

Multicollinearity of independent factors was determined using variance inflation factor and accordingly the factors were retained in the model. As the data included patients with bilateral involvement, in proportional hazard model, patients were treated as clusters with correlated observations to address the interdependency of eyes.

All the computation analysis was performed using SPSS ver 20.0 (IBM Corp. Armonk NY USA) as well as R-3.2.3 (R Foundation, Vienna, Austria) and statistical significance was tested at $5 \%$ level.

\section{Key observations of COTS-1}

This retrospective cohort study included a total of 945 patients from 25 different centers diagnosed with TBU between January 2004 and December 2014. Following are the key observations from the data.

\subsection{Demographic profile of patients}

The mean age of presentation of TBU was found to be $41.3 \pm 15.0$ years (range, 4-90 years) with predominance of males $(52.9 \%, n=500 / 945)$, Asian ethnicity $(74.4 \%, n=$ 690/927) and Asian geographical origin (61.5\%, $n=581 / 927)$; consistent with previous reports. ${ }^{8,9}$ Ninety percent were adults (aged $\geq 15$ years). ${ }^{1}$ One-fifth of the cohort was identified as immigrants $(18.1 \%, n=168 / 927)$ based on the ethnicity foreign to the region 
of recruitment. ${ }^{9}$ Table 1 illustrates demographic profile of patients with TBU. The bias towards the Asian ethnic population and region of origin can be explained by the global burden of TB, as according to WHO Global TB report 2018, in which overall two thirds of TB cases were reported in eight countries, six of which were Asian, with the highest incidence in India (27\%). ${ }^{1}$

\subsection{Systemic disease and ocular TB}

Many prior studies have reported that patients referred with a suspicion of ocular TB lack clinical signs of systemic disease on routine investigations. , $^{3,5,6,8,9,10}$

Of the 787 patients in whom the information was available, only 183 (23.3\%) showed any known history of systemic TB at the time of diagnosis of TBU. ${ }^{9}$ Ocular TB, in fact, represents an extra pulmonary form of the disease, occurring most of the time in isolation, with no clinical or laboratory evidence of pulmonary infection or active disease elsewhere in the body. ${ }^{3,5,6}$ However, the limitation of the retrospective data collection, which lead to a lack of standardization in documentation and hence missing data, need to be considered. A history of pulmonary involvement was found in 129/787 (16.4\%) patients affected, while other non ocular extra pulmonary site involvement accounted for 50/787 (6.35\%). ${ }^{9}$ Given the absence of active associated systemic disease, most of the patients with TBU did not report any symptoms suggestive of active infection $(92.0 \%, n=655 / 712)$ such as significant weight loss ( $\geq 5 \mathrm{~kg} / 6$ months), night sweats, chronic cough and hemoptysis that was reported only in $3.2 \%, 2.5 \%, 2.8 \%$ and $0.4 \%$ of patients respectively. ${ }^{9}$ Data on systemic clinical features and symptoms of patients diagnosed with TBU is shown in Table 2.

\subsection{Anatomic distribution of uveitis}

Bilateral presentation was more common $(n=471 / 801,58.8 \%) .{ }^{10}$ Posterior uveitis was the most frequent clinical presentation in accordance with previous reports. ${ }^{8,10}$ A total of 1246 
eyes were analyzed for the anatomic site of disease involvement. Posterior uveitis was the most common phenotype, found in $452 / 1246$ (36.3\%) patients, followed by panuveitis in 449/1246 (35.3\%), intermediate uveitis in 199/1246 (15.9\%) and anterior uveitis in 155/1246 (12.5\%). ${ }^{10}$ Anatomic distribution of TBU is illustrated in Table 3.

\subsection{Onset}

TBU presented as acute uveitis in 402/873 (48.7\%) patients, with a duration less than six weeks, followed by chronic uveitis ( $\geq 12$ weeks) in $280 / 873$ (33.9\%) and subacute disease (6-12 weeks) in 143/873 (17.3\%) (unpublished data). Characteristics of onset of TBU are shown in Table 3.

\subsection{Clinical features}

Choroidal involvement was found to be the most common clinical sign $(64.4 \%, n=$ $419 / 651)$, followed by vitreous haze $(45.4 \%, n=523 / 1153)$, retinal vasculitis (with occlusive features $-41.4 \%, n=155 / 374$ - and without occlusive features $-31.5 \%, n=$ $118 / 374)$, disc hyperemia or edema $(20.5 \%, n=244 / 1189)$, snow balls $(16.2 \%, n=$ 190/1175), cystoid macular edema $(17.6 \%, n=206 / 1168)$ and snowbanking $(6.1 \%, n=$ 71/1170). ${ }^{10}$ Table 4 illustrates clinical features of patients with TBU. Variable rates of ocular findings and complications have been reported in literature from individual centers, ranging from $9 \%$ to $45 \%$ for choroiditis, $4 \%$ to $61 \%$ for retinal vasculitis, $8 \%$ to $67 \%$ for vitritis, $7.5 \%$ to $45 \%$ for cystoid macular edema, $26 \%$ to $51 \%$ for optic disc involvement, $6 \%$ to $33 \%$ for snowball and $4 \%$ to $8 \%$ for snowbanking. ${ }^{14-22}$

\subsection{Radiologic findings}

Chest computed tomography (CT) was found to be more sensitive in detecting evidence of old healed and inactive TB compared to chest X-ray. ${ }^{9}$ Among the patients with documented radiological results, 189/702 (26.9\%) had features consistent with inactive or 
healed pulmonary TB on chest X-rays and 109/159 (68.6\%) on chest CT scans. ${ }^{9}$ Chest CT scans have thus a valuable diagnostic additional yield in patients with phenotypes suggestive for ocular TB and suspected exposure without active infection. ${ }^{9}$ Results of radiological investigations are detailed in Table 5.

\subsection{Immunological tests}

From the study it has emerged a variable use of tuberculin skin test (TST) and/or either form of immune globulin release assays (IGRAs) among the eye care centers. Overall, 80$90 \%$ of tested patients had a positive immunological result. ${ }^{9}$ The most commonly performed test for ocular TB by participants was TST, that showed positivity in 549/631 (87.0\%) patients, followed by QuantiFERON TB-Gold in-tube (positive in 274/305, 89.9\%) and T-SPOT.TB test (positive in 70/76, 92.1\%). ${ }^{9}$ Results of immunological investigations are illustrated in Table 5.

\subsection{Polymerase chain reaction}

The study assessed the role of polymerase chain reaction (PCR) in the management of ocular TB, analyzing how PCR results are used as a diagnostic criterion and influence the decision on treatment initiation.

Overall, it emerged that PCR is not a common tool in the diagnosis of TBU, since it was performed in a small subset of patients $(n=59 / 962,6.1 \%) .{ }^{11}$

Positive PCR results for Mycobacterium TB were obtained in 33/59 (55.9\%) patients, all Asian Indians. ${ }^{11}$ The bias towards Indian origin of positive results could be explained by the predominantly Asian ethnicity of the patients underwent fluid analysis $(89.8 \%$ of tested patients were Indian). ${ }^{11}$

From the study, it emerged that positive or negative results did not influence management in the real world practice, considering that more than half patients who tested negative 
were diagnosed as TBU and treated with ATT by the uveitis specialist (treatment with ATT was given in $31 / 33$ patients with PCR positive results (93.9\%) compared to 18 patients with PCR negative results $(78.26 \%)) .{ }^{11}$

\subsection{Treatment and global variations in management practices}

COTS-1 highlights differences in TB management practice across the globe. Overall, a total of 801 patients $(n=801 / 947,84.6 \%)$ received ATT, 799 patients $(n=799 / 940$, $85.0 \%)$ corticosteroids and 72 patients $(n=72 / 799,9.0 \%)$ corticosteroid-sparing immunosuppressive agents. ${ }^{9}$ A total of 705 patients $(n=705 / 927,76 \%)$ received both ATT and corticosteroids, 96/927 (10.4\%) received only ATT and 94/927 (10.1\%) received only corticosteroids. ${ }^{9}$ Table 6 details treatment management in ocular TB.

From the study emerged large heterogeneity in the usage of individual treatment modalities based on geographic distribution. The proportion of patients who received treatment with both ATT and corticosteroids was higher in East/Asia ( $n=463 / 576,80.4 \%$ ) and Middle-East $(n=64 / 76,84.2 \%)$, compared to Australia $(n=27 / 45,60.0 \%)$ and the West $(n=151 / 240,62.9 \%)$, with the limitation of no centers from sub-Saharan Africa. ${ }^{9}$ Proportion of patients treated only with corticosteroids was lowest in East/Asia $(n=$ $69 / 576,12.0 \%)$ and variable between $5.3 \%(n=4 / 76)$ in Middle-East, $6.7 \%(n=3 / 45)$ in Australia and $7.5 \%(n=18 / 240)$ in the West. ${ }^{8}$ Percentage of patients treated with only ATT was higher in Australia ( $n=15 / 45,33.3 \%)$ compared to the West $(n=47 / 240,19.6 \%)$, Middle-East $(n=8 / 76,10.5 \%)$ and East/Asia $(n=26 / 57,4.5 \%) .{ }^{9}$ Refer to Table 6 for global variation in management practices. Regional differences in treatment practices can be explained by the absence of internationally accepted guidelines among uveitis experts regarding treatment of ocular TB, including the concurrent use of oral corticosteroids and immunomodulatory therapy. It has thus emerged that globally most of the patients with ocular TB are treated with a combination of ATT and corticosteroids, with physicians 
coming from Australia and the West less incline to add corticosteroids to ATT. ${ }^{9}$ A limitation of this study was the retrospective method, which led to a lack of standardization in the documentation among the centers and hence to the lack of data, such as the exact duration of follow-up or the ATT used, which are therefore not described.

\subsection{Treatment outcome in patients with ocular TB treated with ATT}

Several studies have observed a greater proportion of successful outcomes in patients with ocular TB treated with ATT compared to those who did not undergo ATT therapy. ${ }^{8}$ In this study treatment failure was defined as patients with persistence or recurrence of inflammation within 6 months of completing ATT in the involved eyes for patients with unilateral disease or in either eye for patients with bilateral disease, or inability to taper oral corticosteroids to less than $10 \mathrm{mg} / \mathrm{d}$ or corticosteroid eye drops to less than 2 drops daily, or recalcitrant inflammation that necessitated corticosteroid-sparing immunosuppressive therapy. ${ }^{10}$ Our study confirmed a low rate of treatment failure $(12.7 \%)$ in patients with TBU treated with ATT. ${ }^{10}$ From COTS-1 data, it has emerged that presence of choroidal involvement with vitreous haze and snow banking in patients with panuveitis is associated with a higher risk of treatment failure. ${ }^{10}$ This findings are consistent with the current literature that patients having intermediate uveitis or panuveitis had higher odds of recurrences. ${ }^{23}$ The hypothesis for the poor response to ATT in patients with choroidal involvement and vitreous haze could be that certain phenotypes may represent ocular manifestations of autoimmune reaction to molecular mimicry of latent $T B$, instead of active ocular TB infection, and thus they require a more aggressive immunosuppressive treatment. The rational for this hypothesis is that M.Tuberculosis spread from systemic sites of primary infection, such as the lung, via vasculature and reached the eye, where could incite an immune-mediated hypersensitivity reaction, leading to choroiditis. 
In contrast to a recent meta-analysis that reported no significant difference in treatment outcomes in patients with TBU treated with ATT with or without systemic corticosteroids, our study identified a poorer outcome in patients treated with concomitant systemic corticosteroid therapy. ${ }^{8,10}$ This finding could be contributed by the group that received systemic corticosteroids before the initiation of ATT, consistent with the existing doctrine that physicians should delay initiation of systemic corticosteroids until after starting ATT in patients with clinical suspicion of infectious disease, unless there is a high risk of complications secondary to intense inflammatory reaction.

Our data also highlighted the variations in the treatment outcomes between different ethnicity and geographic regions of origin. ${ }^{9}$ Treatment outcomes were superior in Middleeastern or Asian ethnicity, Australian or Asian geographical region of recruitment and nonimmigrants. ${ }^{9}$ The finding of poorer outcomes characterizing patients recruited in the West compared to those from the East may be attributable to the lower epidemiologic burden of TB in Western countries and a lower likelihood of TB as a cause of uveitis. Migrants and ethnic minorities have always been identified as a subpopulation with higher risk for TB compared with the general population..$^{5,24}$ Our findings confirm this hypothesis also in relation to ocular tuberculosis, identifying that patients with African and Hispanic ethnicity and positive immigrant status had a poorer survival outcome. ${ }^{9}$ No age or sex-related variations in treatment outcome were found in our cohort of patients. ${ }^{9}$

The worst treatment outcome was reported in patients that did not receive any treatment i.e., ATT or oral corticosteroid. ${ }^{9}$ Stratifying the analysis for region of origin, patients coming from Asia receiving no treatment and those from the West and the Middle East treated with corticosteroids before initiating ATT had the poorest outcome. ${ }^{9}$ The finding of patients receiving systemic corticosteroids before initiation of ATT affected by distinctly high incidence of treatment failure could indicate a delay in the diagnosis of ocular TB with 
multiple recurrences as the specific therapy could not be initiated and this would result in worse outcome. On the contrary, patients coming from Asia treated with corticosteroids prior the initiation of ATT had a greater successful outcome compared to those receiving steroids during or after ATT or treated without them. ${ }^{9}$ This finding might be attributable to the geographical variability of TB phenotypes. ${ }^{12,13}$

The study thus attributes to ethnicity, geographical origin and immigrant status a role in determining the treatment outcome of patients affected by ocular TB, with African or Hispanic race, patients coming from the West and positive immigrant status significantly associated with treatment failure. ${ }^{9}$

\section{Specific phenotypes studied in COTS-1}

\subsection{Tubercular retinal vasculitis}

The subset analysis of the clinical features of 251 patients diagnosed with tubercular retinal vasculitis (TRV) confirmed the previously reported findings that vasculitis due to TB is predominantly occlusive in nature (features of occlusive type of TRV $n=113 / 185$;

$61.1 \%) .{ }^{13,25}$ However the study revealed a lower frequency of occlusive features in Caucasians (28.2\%), compared to other races (71.6\% in Asian, $63.6 \%$ in Middle-Eastern population, $61.5 \%$ in black Africans and $50 \%$ in Hispanics). ${ }^{13}$ The same trend is reflected on geographical distribution of TRV phenotype ( $51.3 \%$ of occlusive TRV in the West, $60.0 \%$ in Middle-East and $67.8 \%$ in the East), with the exception of Australia, where, although $56.4 \%$ of population report a European ancestries, occlusive features accounted for $87.5 \%$ of TRV. ${ }^{13,26}$ Global variation in distribution of TRV phenotype is detailed in Table 7.

There was no significant difference in treatment failure whether patients received ATT, although, in all geographical regions, treatment failure was less frequent in patients 
receiving ATT or ATT and steroids ( $n=31 / 228,13.6 \%$ ), compared with those who did not $(n=5 / 23,21.7 \%) .{ }^{13}$ Among patients receiving ATT, those with occlusive features had better prognosis. ${ }^{13}$

ATT therapy was associated with higher failure rates in Hispanic and African patients with TRV, and, according to the study data on treatment outcome in patients with TBU treated with ATT, in patients with TRV associated with panuveitis. ${ }^{13}$

\subsection{Tubercular choroiditis}

From the analysis of choroidal involvement in 245 patients with TBU has emerged that serpiginous-like choroiditis (SLC) was the most common phenotype ( $n=113 / 245,46.1 \%$ ), followed by choroidal tuberculoma $(n=33 / 245,13.5 \%)$ and multifocal choroiditis (MFC) ( $n$ $=23 / 245,9.4 \%) .{ }^{12}$ Other phenotypic variants considered rare as TB manifestation such ampiginous choroiditis (APC) or acute posterior multifocal placoid pigment epitheliopathy (APMPPE) were observed in 22/245 (9.0\%) and 8/245 (3,3\%), respectively. ${ }^{12}$ Unclassified variants of phenotype accounted for $46 / 245$ (18.8\%) of choroidal involvement. ${ }^{12}$ Phenotypical expression of choroidal involvement is given in Table 7. Choroidal involvement was not commonly associated with other structural involvement, since vitreous haze, snow balls, snow banking, disc hyperemia and macular edema were absent in more than $70 \%$ of cases. ${ }^{12}$

SLC was the most prevalent phenotype in patients coming from Asia, Australia and Middle-East, described in $56.0 \%, 60.0 \%$ and $40.5 \%$ of choroidal involvement, respectively, and less frequent in the West (25.8\%). Here the most common form was unclassified variants, accounted for $29.0 \%$ of phenotypic expressions (information is shown in Table 7). ${ }^{12}$

The study provided the first description of geographical variation in TB choroiditis, revealing how phenotypes infrequently associated with TB as APC or APMPPE account 
for more than $10 \%$ of global choroidal variants. ${ }^{12}$ Most of the patients were treated with a combination of ATT and steroids $(n=220 / 245,85.7 \%) .{ }^{12}$ A variability in the responsiveness of different phenotypes emerged and identified the lower treatment failure in patients with APC and SLC. ${ }^{12}$

\section{Conclusions and Summary}

COTS-1 is the largest collaborative multicenter data set on TBU from multiple leading international eye care centers, provided a global overview of ocular TB. Results from COTS-1 suggest a lack of comprehensive evidence for diagnostic and therapeutic approaches in ocular TB, with no internationally accepted management protocol and regional variations in the workup of patients supposedly affected. COTS-1 also identified regional differences in the frequencies of some typical phenotypes, the reason for which remains to be determined.

A limitation of this study is the retrospective methods, which leads to a lack of standardization in documentation and hence missing data. Selection bias can arise, given the diagnostic ambiguity in some cases, and there is a lack of a standardized systematic way to identify all patients with TBU over a 10-year period retrospectively. Additionally, there were no centers from sub-Saharan Africa due to lack of collaborators. Another limitation is that some clinical information was not available for the study. Regardless of these limitations, this study had strengths of standardized inclusion and diagnostic criteria and a large and diverse cohort of patients.

Diagnostic and management uncertainties highlighted by the study raise the need for future prospective studies to better assess ocular TB clinical features and to enhance the study of management and treatment outcomes of ocular TB through standardized recruitment and treatment protocol. To date, there are no randomized controlled trials that 
have defined the diagnosis nor the management of ocular TB. Furthermore, there are no consensus guidelines among the uveitis experts regarding the treatment of ocular TB, including duration of ATT, ATT regime and also regarding the concurrent use of oral corticosteroids and immunomodulatory therapy (either systemic or local). In addition, there is no multicenter study on TB that highlights differences in practice across the globe. These observations underscore the need for multinational prospective data collection and analysis in collaboration with pulmonary and/or infectious disease specialists to directly manage and address these uncertainties.

We have now embarked on the Collaborative Ocular Tuberculosis Study Consensus (COTS CON), an international, expert led consensus initiative with the aim of developing systematic, evidence and experience-based recommendations for the treatment of ocular TB, consolidating the expertise of international uveitis specialists on an accepted approach to the management of ocular TB. A clinical risk score with standardized assessment of phenotypes and investigation results might be of value to address these issues and determine the role of ATT in patients with ocular TB. 


\section{References}

1. World Health Organization (WHO) Global Tuberculosis Report 2018. www.who.int/tb/publications/global_report/en/

2. Abu El-Asrar AM, Abouammoh M, Al-Mezaine HS. Tuberculous uveitis. Int Ophthalmol Clin. 2010;50:19-39.

3. Lee C, Agrawal R, Pavesio C. Ocular Tuberculosis - A clinical conundrum. Ocul Immunol Inflamm. 2016;24(2):237-42.

4. Ang M, Chee SP. Controversies in ocular tuberculosis. Br J Ophthalmol. 2017;101(1):6-9.

5. Gupta V, Gupta A, Rao NA. Intraocular tuberculosis - an update. Surv Opthalmol. 2007;52(6):561-87

6. Gupta A, Sharma A, Bansal R, Sharma K. Classification of intraocular tuberculosis. Ocul Immunol Inflamm. 2015;23(1):7-13.

7. Rosenbaum JT. To be or not TB? Br J Ophthalmol. 2014;98(8):999-1000.

8. Kee AR, Gonzalez-Lopez JJ, Al-Hity A et al. Anti tubercular therapy for intraocular tuberculosis: A systematic review and meta-analysis. Surv Ophthalmol. 2016;61(5):628-53.

9. Agrawal R, Gunasekeran DV, Raje D, et al. Global variations and challenges with Tubercular uveitis in the Collaborative Ocular Tuberculosis Study. Invest Ophthalmol Vis Sci. 2018;59(10):4162-4171.

10. Agrawal R, Gunasekeran DV, Grant R, et al. Clinical features and outcomes of patients with tubercular uveitis treated with antitubercular therapy in the Collaborative Ocular Tuberculosis Study (COTS)-1. JAMA Ophthalmol. 2017;135(12):1318-1327.

11. Agarwal A, Agrawal R, Gunasekaran DV, et al. The Collaborative Ocular Tuberculosis Study (COTS)-1 Report 3: Polymerase chain reaction in the diagnosis and management of tubercular uveitis: global trends. Ocul Immunol Inflamm. 2017;00:1-9. 
12. Agarwal R, Gunasekaran DV, Agrawal A, et al. The Collaborative Ocular Tuberculosis Study (COTS)-1: a multinational description of the spectrum of choroidal involvement in 245 patients with tubercular uveitis. Ocul Immunol Inflamm. 2018;29:1-11.

13. Gunasekeran DV, Agrawal R, Agarwal A, et al. The Collaborative Ocular Tuberculosis Study (COTS)-1: A Multinational Review of 251 Patients With Tubercular Retinal Vasculitis. Retina. 2018

14. Al-Qarni A, Abouammoh MA, Almousa AN, Mousa A, Abu El-Asrar AM. Presumed tuberculous uveitis in a university-based tertiary referral center in Saudi Arabia. Int Ophthalmol. 2019;39(2):317-333.

15. Ang L, Kee A, Yeo TH, et al. Treatment outcome in patients with presumed tubercular uveitis at a tertiary referral eye care centre in Singapore. Int Ophthalmol. 2018;38(1):11-18.

16. Manousaridis K, Ong E, Stenton C, Gupta R, Browning AC, Pandit R. Clinical presentation, treatment, and outcomes in presumed intraocular tuberculosis: experience from Newcastle upon Tyne, UK. Eye (Lond). 2013;27(4):480-486.

17. Ng KK, Nisbet M, Damato EM, Sims JL. Presumed tuberculous uveitis in non-endemic country for tuberculosis: case series from a New Zealand tertiary uveitis clinic. Clin Experiment Ophthalmol. 2017;45(4):357-365.

18. Vos AG, Wassenberg MWM, de Hoog J, Oosterheert JJ. Diagnosis and treatment of tuberculous uveitis in a low endemic setting. Int J Infect Dis. 2013;17(11):e993-999.

19. Khochtali S, Gargouri S, Abroug N, et al. The spectrum of presumed tubercular uveitis in Tunisia, North Africa. Int Ophthalmol. 2015;35(5):663-671.

20. Gupta A, Bansal R, Gupta V, Sharma A, Bambery P. Ocular signs predictive of tubercular uveitis. Am J Ophthalmol. 2010;149(4):562-570.

21. Shahidatul-Adha M, Zunaina E, Liza-Sharmini AT, et al. Ocular tuberculosis in Hospital Universiti Sains Malaysia - A case series. Ann Med Surg (Lond). 2017;24:25-30.

22. La Distia Nora R, van Velthoven MEJ, Ten Dam-van Loon NH, et al. Clinical manifestations of patients with intraocular inflammation and positive QuantiFERON-TB gold in-tube test in a country nonendemic for tuberculosis. Am J Ophthalmol. 2014;157(4):754-761. 
23. Agrawal R, Gupta B, Gonzalez-Lopez JJ. The role of anti-tubercular therapy in patients with presumed ocular tuberculosis. Ocul Immunol Inflamm. 2015;23(1):406.

24. Hayward S, Harding RM, McShane $H$ and Tanner R. Factors influencing the higher incidence of tuberculosis among migrants and ethnic minorities in the UK. F1000Res. 2018;7:461.

25. Gupta V, Shoughy SS, Mahajan S, et al. Clinics of ocular tuberculosis. Ocul Immunol Inflamm. 2015;23(1):14-24.

26. Census of Population and Housing: Census Dictionary, 2016 (cat. no. 2901.0). www.abs.gov.au/census. 


\section{Appendix 1}

Patients need to satisfy both criteria 1 and 2 along with either 3 or 4 to be recruited in the COTS-1:

1. Any of the following clinical signs suggestive of IOTB, including:

a. Anterior uveitis (granulomatous or non-granulomatous) with or without iris nodules or ciliary body granuloma or

b. Intermediate uveitis (granulomatous or non-granulomatous with exudates in the pars plana or peripheral uvea with snow balls) or

c. Posterior or panuveitis - choroidal tubercle or choroidal granuloma or subretinal abscess or serpiginous-like choroiditis or retinitis or retinal vasculitis or

d. Neuroretinitis or optic neuritis or endogenous ophthalmitis or panophthalmitis or scleritis;

2. Exclusion of other uveitic entities where relevant, based on clinical manifestations of disease and regional epidemiology;

3. Investigations documenting the mycobacteria or its genome:

a. Demonstration of Acid-Fast Bacilli (AFB) by microscopy or culture of M. tuberculosis from ocular fluid,

b. Positive polymerase chain reaction from ocular fluid for IS 6110 or other conserved sequences in mycobacterial genome,

c. Evidence of confirmed active pulmonary or extrapulmonary tuberculosis (by microscopic examination or culture of a tissue sample from the affected tissue);

4. Corroborative investigations:

a. Positive Mantoux reaction (must be accompanied by information regarding antigen and amount of tuberculin injected, along with institutional practices in 
interpreting the test),

b. Interferon Gamma Release Assay (IGRA) such as Quantiferon TB Gold (must be accompanied by information regarding institutional practices in interpreting the test),

c. Evidence of healed or active tuberculosis on chest radiography (must be accompanied by information regarding practices by institution radiologists regarding clinical features that are considered evidence in this regard). 